\title{
SHOCK SOLUTIONS FOR PARTICLE-LADEN THIN FILMS
}

\author{
BENJAMIN P. $\mathrm{COOK}^{\dagger}$, ANDREA L. BERTOZZI ${ }^{\dagger}$, AND A. E. HOSOI ${ }^{\ddagger}$
}

\begin{abstract}
We derive a lubrication model describing gravity-driven thin film flow of a suspension of heavy particles in viscous fluid. The main features of this continuum model are an effective mixture viscosity and a particle settling velocity, both depending on particle concentration. The resulting equations form a $2 \times 2$ system of conservation laws in the film thickness $h(x, t)$ and in $\phi h$, where $\phi(x, t)$ is the particle volume fraction. We study flows in one dimension under the constant flux boundary condition, which corresponds to the classical Riemann problem, and we find the system can have either double-shock or singular shock solutions. We present the details of both solutions and examine the effects of the particle settling model and of the microscopic length scale $b$ at the contact line.
\end{abstract}

Key words. thin liquid films, contact lines, Riemann problem, systems of conservation laws, suspension flow, sedimentation, singular shocks

AMS subject classifications. 76D08, 76D07, 35L65, 76T20

1. Introduction. The flow of thin viscous films [44] is of great importance to many problems in science and engineering. Flows in thin films can result from slow processes such as spreading [17] and evaporation [6], or stronger driving forces such as capillarity [38] or gravity [21]. As shown by Huppert in [21], gravity-driven films on an incline can be described roughly by the conservation law

$$
\frac{\partial h}{\partial t}+\frac{\partial}{\partial x} h^{3}=0
$$

for the film thickness $h$. However, in many cases, large gradients in $h$ and dry regions where $h=0$ exist, requiring more complex models that incorporate capillary forces and the thermodynamic wetting process.

Wetting occurs as a fluid domain evolves, moving in particular the contact line, where the solid, liquid, and vapor phases meet. Despite the fundamental importance of contact lines to fluid dynamic boundary conditions, many of their basic properties are not fully understood $[2,13]$. The standard no-slip boundary condition is inadequate near a moving contact line [14,20], and two common contact line models either allow a small slip velocity [20] or assume a thin "precursor" film rather than a dry substrate [55]. These models have contributed to understanding of the capillary ridge that often develops near the contact line $[3,16,19,23,28,55]$, the rupture of thin films [39, 57], the contact angle that the free surface makes with the substrate $[16,23,25,50]$, and the relevance of the material composition of the fluid and substrate $[12,24,50]$. A "fingering" instability observed in [21] which deforms the contact line in some driven films has also motivated analysis [3, 55], simulation [28], and experiments [12, 24, 50] on thin film flow.

Film flows of more complex materials are much less understood. For dry granular flows, air can frequently be neglected, and the central modeling challenge is to determine an appropriate constitutive relation [26]. Replacing the fluid with a suspension,

${ }^{\dagger}$ Department of Mathematics, University of California Los Angeles, Los Angeles, California. These authors were supported by NSF VIGRE grant DMS-0502315, NSF grant ACI-0321917, ONR grant N000140410078, NSF grant DMS-0244498

${ }^{\ddagger}$ Hatsopoulos Microfluidics Laboratory, Department of Mechanical Engineering, Massachusetts Institute of Technology, Cambridge, Massachusetts. This author was supported by NSF grant 0323672 
however, introduces the possibility of phase segregation, allowing new behaviors not seen in pure fluids and only recently observed in film flows [54, 59]. Segregation of viscous suspensions can be driven by gravity [59], but has also been observed with neutrally buoyant particles in thin films [54] and in the related Hele-Shaw flow [53]. Direct numerical simulation of suspension flows considering individual particles is computationally demanding and existing methods do not account for the complexities of a contact line [49,58]; consequently, continuum models play an essential role in understanding these flows.

Continuum descriptions of viscous suspensions involve three main effects: an "effective viscosity" greater than that of the suspending fluid $[30,52]$, the settling of heavy particles due to gravity [11], and particle fluxes thought to result from particle interactions in the presence of shear [34]. Various models have incorporated some or all of these effects $[40,42,45,47]$, however only a limited number of flow geometries have been studied, most commonly the one-dimensional Couette and Poiseuille flows (for exceptions see [15]). In particular Schaflinger et al. [47] model a gravitydriven thin film, though they do not consider variation in the flow direction caused by gravitational segregation that we will model below.

Our work is motivated by the experiment and model described by Zhou et al. in [59]. The experiment consists of a gravity-driven film of a dense ( $\geq 17 \%$ by volume) suspension of glass beads in oil which flows down an inclined plane under constant flux upstream conditions. They observed three different particle behaviors in this experiment, depending on the inclination angle and particle concentration of the initially well-mixed suspension, which they summarized in a phase diagram. At low inclination angles and concentrations, the particles settle out of the flow leaving a film of clear fluid, while at intermediate angles and concentrations the suspension appeared well-mixed for the duration of the experiment. At high angles and concentrations the particles accumulate near the moving contact line in a "particle-rich ridge". They also observed that while the well-known fingering instability [21] occurs in the first two regimes, it is largely suppressed when the ridge appears. Their new model describes this third regime, characterized by spatially varying rheology, which appears to have no analogue in pure fluid motion.

Zhou et al. derived their model by treating the mixture locally as a Newtonian fluid, which allows the use of standard lubrication techniques. The two-phase flow is described by an overall velocity determined from the local value of a concentrationdependent effective viscosity, and a settling velocity of the heavy particles relative to the fluid. They derived a system of two coupled fourth-order evolution equations for the film thickness and particle concentration, and argued that the essential dynamics are determined by a system of conservation laws obtained by retaining only the firstorder terms. They also presented double-shock solutions for this system depending on the parameter $b$ appearing in their contact line model, which represents the thickness of a precursor film appearing ahead of the bulk flow. They compared these solutions to the experimentally observed ridge, and noted that the calculated speeds of the two shocks become nearly equal at small values of $b$. Their calculations however were not sufficient to determine whether the shock speeds actually coincide at some finite $b^{*}>0$, an important issue as this would call into question the existence of solutions for $b<b^{*}$. Furthermore, they described the physical derivation and the shock solutions only briefly.

The purpose of the present work is to give a more complete derivation of this model and to more thoroughly characterize its shock solutions, including their dependence 
on $b$. In $\S 2$ we present a full derivation following the assumptions of Zhou et al. While the equations we derive are slightly different, they appear to have the same qualitative behavior. In $\S 3$ we recall the classical theory for hyperbolic systems of conservation laws, and in $\S 4$ we apply these methods to the system we have derived. For double shock solutions, we find the two shock speeds do become equal at a certain precursor thickness $b$, below which the equations have no classical solution. In $\S 5$ we compare this case to the mathematical theory of singular shocks, in which a delta mass is concentrated at the discontinuity. We study one approximate singular shock solution and find the particle concentration exceeds the limit of close packing, suggesting the model is inaccurate at high concentrations. We propose a modified form for the settling velocity in $\S 6$ which causes both the particle and fluid velocities to vanish at close packing, and find the resulting equations appear to be well-posed for all precursor thicknesses. Then we conclude in $\S 7$ that the modified equations represent a more realistic model for particle-laden films.

2. Derivation. Two common methods for describing binary mixtures in a continuum framework are the "two-fluid" and the "mixture" or "one-fluid" models [56]. The two-fluid model balances forces on the two components separately, with the forces of interaction appearing explicitly as a function of the two velocities. It therefore requires a separate viscosity for each phase. The one-fluid model balances forces on the mixture as a whole, using an effective viscosity, and postulates a form for the relative velocity between the two components. Since empirical formulae are readily available for the effective mixture viscosity and settling velocity, we follow Zhou et al. in using the one-fluid model. We also note that the fluid and particle velocities are nearly equal, so the one-fluid equations describing average and relative velocities can be expected to be less strongly coupled than their two-fluid counterparts.

Deriving a one-fluid model involves balancing forces first for the mixture as a whole, without regard to interactions between the two components. In the present case inertia is negligible, and these forces are just gravity and viscous stress. We use an empirical expression for the latter in which the mixture is considered a Newtonian fluid, with an effective viscosity depending on the particle volume fraction $\phi$. For a fluid of kinematic viscosity $\mu_{f}$ one form for this relation is $[30,52]$

$$
\mu(\phi)=\mu_{f}\left(1-\phi / \phi_{m}\right)^{-2},
$$

where $\phi_{m} \approx 0.67$ is the random packing fraction of spheres. This viscosity leads to a stress tensor of the form

$$
\mathbf{\Pi}=p \mathbf{I}-\frac{1}{2} \mu(\phi)\left[\nabla \mathbf{v}+(\nabla \mathbf{v})^{T}\right]
$$

where $p$ is the fluid pressure and $\mathbf{v}$ is a velocity characterizing the motion of the mixture. Since the two mixture components in general have different velocities, say $v_{f}$ and $v_{p}$ for the fluid and particulate phases respectively, $\mathbf{v}$ must be some average of the two. Much of the experimental literature deals with neutrally buoyant mixtures, in which the two velocities are the same and the distinction is unnecessary, but in the current case the question is relevant. We argue that since the effective viscosity phenomenon involves neither inertia nor gravity, it should be independent of the masses of the two phases, therefore we select the volume-averaged velocity. Defining

$$
\mathbf{v}=(1-\phi) \mathbf{v}_{\mathbf{f}}+\phi \mathbf{v}_{\mathbf{p}}, \quad \mathbf{v}_{\mathbf{r e l}}=\mathbf{v}_{\mathbf{p}}-\mathbf{v}_{\mathbf{f}}
$$


thus comprises the one-fluid model for the mixture, and if $\phi$ is known the individual phase velocities can be recovered by

$$
\mathbf{v}_{\mathbf{p}}=\mathbf{v}+(1-\phi) \mathbf{v}_{\text {rel }}, \quad \mathbf{v}_{\mathbf{f}}=\mathbf{v}-\phi \mathbf{v}_{\text {rel }} .
$$

The average velocity satisfies the Stokes equations:

$$
\nabla \cdot \boldsymbol{\Pi}=\rho(\phi) \mathbf{g}, \quad \nabla \cdot \mathbf{v}=0,
$$

where $\rho(\phi)$ is the mixture density and $\mathbf{g}$ is the gravitational field. The density is given by $\rho(\phi)=\rho_{f}(1+\Delta \phi)$, where $\Delta=\left(\rho_{p}-\rho_{f}\right) / \rho_{f}$ and $\rho_{f}$ and $\rho_{p}$ are the densities of the fluid and particulate phases.

We now define the problem geometry, considering a plane solid surface inclined at an angle $\theta$ to the horizontal, to be coated with an advancing gravity-driven film. We choose a coordinate system in which $\mathbf{z}$ is normal to the inclined plane, $\mathbf{x}$ and $\mathbf{y}$ lie in the inclined plane, $\mathbf{y} \cdot \mathbf{g}=0$, and suppose the film emerges from a gate at $x=0$ at a constant thickness and particle concentration for $t>0$.

In deriving the equation for $\mathbf{v}$, we follow the standard methods used for pure fluid films $[17,44]$. The lubrication approximation, valid at small Reynolds numbers and geometric aspect ratios, assumes $\mathbf{v}$ lies in the $\mathbf{x}-\mathbf{y}$ plane and $\left|\frac{\partial \mathbf{v}}{\partial z}\right| \gg \max \left(\left|\frac{\partial \mathbf{v}}{\partial x}\right|,\left|\frac{\partial \mathbf{v}}{\partial y}\right|\right)$. Correspondingly, we now consider all velocities to be two-dimensional vectors, as well as the gradient $\nabla=\mathbf{x} \frac{\partial}{\partial x}+\mathbf{y} \frac{\partial}{\partial y}$, and define $g_{\perp}=\mathbf{g} \cdot \mathbf{z}=|\mathbf{g}| \cos \theta$ and $\mathbf{g}_{\|}=\mathbf{g}-g_{\perp} \mathbf{z}=$ $(|\mathbf{g}| \sin \theta) \mathbf{x}$. In this notation, the Stokes equations now read

$$
\begin{array}{r}
\frac{\partial p}{\partial z}=-\rho(\phi) g_{\perp}, \\
\nabla p=\mu(\phi) \frac{\partial^{2} \mathbf{v}}{\partial z^{2}}+\rho(\phi) \mathbf{g}_{\|} .
\end{array}
$$

The Laplace-Young boundary condition states that the pressure at the free surface, $z=h(x, y)$, is given by

$$
p(x, y, h(x, y))=-\gamma \nabla^{2} h(x, y)
$$

where $\gamma$ is the coefficient of surface tension. The pressure is then determined by

$$
p(x, y, z)=-\gamma \nabla^{2} h(x, y)+\int_{z}^{h(x, y)} \rho\left(\phi\left(x, y, z^{\prime}\right)\right) g_{\perp} d z^{\prime}
$$

from the depth and particle concentration of the film. Here it is convenient to assume the particle concentration is independent of the $z$ coordinate, so that the integral in $(2.8)$ is merely $\rho(\phi) g_{\perp}(h-z)$. We will discuss this assumption further in our treatment below of particle motion.

Combining (2.6b) and (2.8) and defining $P(x, y)=-\gamma \nabla^{2} h+\rho(\phi) g_{\perp} h$, we have

$$
\nabla P-z g_{\perp} \nabla \rho=\rho(\phi) \mu(\phi) \frac{\partial^{2} \mathbf{v}}{\partial z^{2}}+\rho(\phi) \mathbf{g}_{\|} \cdot
$$

The boundary conditions of interest are no stress $(\partial \mathbf{v} / \partial z=0)$ at the free interface and no slip $(\mathbf{v}=0)$ at the solid interface. Equation (2.9) can now be integrated twice in $z$ with the constants of integration determined by these conditions, to arrive at the equation

$$
\mu(\phi) \mathbf{v}=\left(h z-\frac{z^{2}}{2}\right)\left(\rho(\phi) \mathbf{g}_{\|}-\nabla P\right)+\frac{1}{2}\left(h^{2} z-z^{3} / 3\right) g_{\perp} \nabla \rho(\phi)
$$


for the volume-averaged velocity. Integrating once more gives the depth-averaged velocity

$$
\mathbf{v}_{\mathbf{a v}}=\frac{h^{2}}{3 \mu(\phi)}\left[\gamma \nabla \nabla^{2} h-g_{\perp}\left(\nabla(\rho(\phi) h)-\frac{5}{8} h \nabla \rho(\phi)\right)+\rho(\phi) \mathbf{g}_{\|}\right] .
$$

Modeling the relative velocity due to particle settling turns out to be more difficult. Recall that in the above lubrication analysis, we have assumed the particles are evenly distributed across the film depth. This may seem unrealistic because the normal component of gravity is pulling the particles toward the solid substrate, but Zhou et al. found that particles settle out of the fluid only at low angles and concentrations. Additionally a thin film experiment done by Timberlake and Morris with neutrally buoyant particles found higher concentrations near the free surface [54]; they attributed this to a shear-induced particle flux such as Leighton and Acrivos describe in [34]. This flux consists of a nonlinear diffusion in the presence of shear, and in inhomogeneous flows an additional migration of particles away from regions of high shear. Schaflinger et al., in their model for film flow [47], balance gravity-driven settling with only the diffusive flux, and only achieve steady state when concentration increases with depth. While the corresponding problem including both diffusion and migration remains unsolved, Carpen and Brady found non-monotone concentration profiles in a model for the related inclined Poiseuille flow [9], and also showed these profiles are unstable due to heavy material suspended above lighter material. Thus it is unclear even whether the actual concentration profile for film flow increases or decreases with depth, and since we are seeking a simple model we find the uniform depth profile to be reasonable.

We begin our model of the relative motion with the settling velocity

$$
\mathbf{v}_{\mathbf{s}}=\frac{2 a^{2} \Delta \rho_{f} \mathbf{g}_{\|}}{9 \mu_{f}}
$$

of a heavy sphere in $\mathbb{R}^{3}$, while noting that this expression neglects the effects of the solid boundary, the free surface, and other particles. The problem of determining settling rates of concentrated mixtures is complex, even in the idealized case of monodisperse spherical particles in a large domain [11]. Some of the challenges are summing the interactions between spheres, which decay only as $1 / r$ in Stokes flows, and interpreting theoretical results that imply divergent fluctuations about the mean particle velocity $[5,8]$. Since there is no general agreement of theoretical and numerical results with experiments, sedimentation is commonly modeled by the empirical hindered settling function

$$
\mathbf{v}=f_{R Z}(\phi) \mathbf{v}_{\mathbf{s}}=(1-\phi)^{n} \mathbf{v}_{\mathbf{s}}
$$

where $n \approx 5$ for Stokes flow [46].

We also seek a correction to represent the impeding effect of the solid substrate on particle motion. The Stokes problem for a sphere settling in a half-space parallel to a wall has been solved approximately by the method of images [18], leading to the series solution

$$
\mathbf{v}=\left(1-\frac{259}{256}\left(\frac{a}{h}\right)+\frac{9}{16}\left(\frac{a}{h}\right) \log \left(\frac{a}{h}\right)-\frac{1}{16}\left(\frac{a}{h}\right)^{3}+\frac{15}{256}\left(\frac{a}{h}\right)^{4}+\ldots\right) \mathbf{v}_{\mathbf{s}}
$$

for the velocity, where $h>a$ is the distance from the center of the particle to the wall. Since we seek a depth-averaged solution we are concerned not with the velocity 
itself, but its average value over the interval $(0, h)$. Figure 2.1 shows this average for a range of $h / a$, where the velocity has been taken to be zero for $h<a$. Also plotted is the correction we will use to approximate wall effects:

$$
w(h)=\frac{A(h / a)^{2}}{\sqrt{1+\left[A(h / a)^{2}\right]^{2}}}
$$

with $A=1 / 18$. This function has the desired properties $w \approx 0$ for $h<a, w \approx 1$ for $h \gg a$, and unlike equation (2.14) is differentiable and positive on $(0, \infty)$. We have chosen the parameter $A$ so that this function resembles (2.14), but since the latter neglects the net flow and the effects of other particles it should mainly be viewed as a correction to ensure $\mathbf{v}_{\text {rel }} \rightarrow 0$ for very thin films.

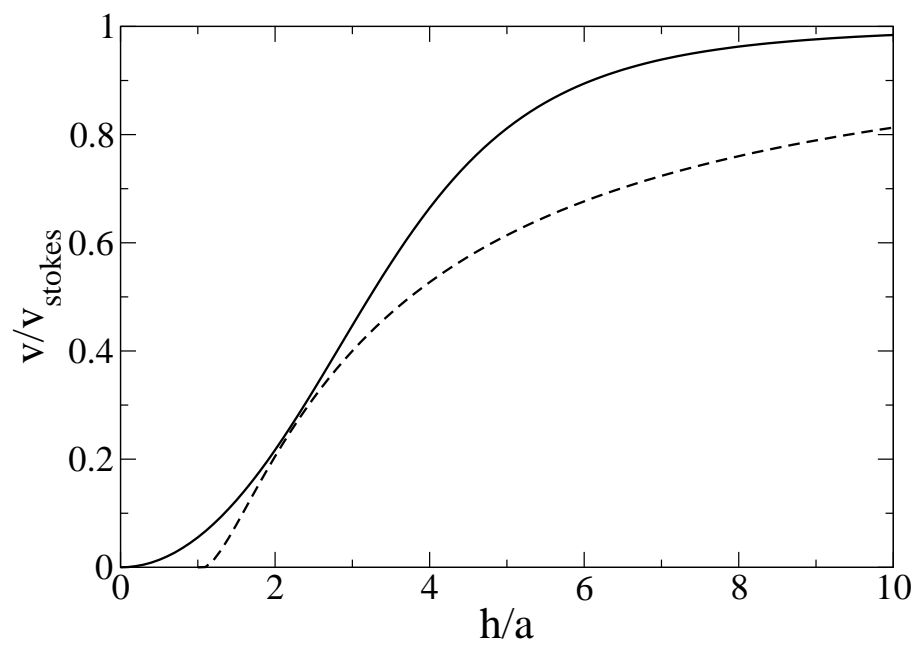

FIG. 2.1. Our correction for the impeding effect of the solid boundary on a single particle's settling velocity (solid), and an analytical result neglecting the free surface and large-scale fluid motion (dashed).

For lack of a comprehensive theory incorporating both wall effects and hindered settling, we simply assume the effects are multiplicative, obtaining the settling velocity

$$
\mathbf{v}_{\mathbf{r e l}}=f(\phi) w(h) \mathbf{v}_{\mathbf{s}}
$$

relative to the fluid which we interpret as a depth average. Having specified the two velocities required for a one-fluid description, we apply conservation of volume to the mixture as a whole and separately to the particulate phase, obtaining the evolution equations:

$$
\frac{\partial h}{\partial t}+\nabla \cdot\left(h \mathbf{v}_{\mathbf{a v}}\right)=0, \quad \frac{\partial \phi h}{\partial t}+\nabla \cdot\left(\phi h \mathbf{v}_{\mathbf{p}}\right)=0 .
$$

Note that (2.17) differs from the model proposed by Zhou et al. Since we use a volume averaged velocity, we apply conservation to volume rather than mass. Inserting equations (2.11), (2.16), and (2.4) into (2.17) now gives us the complete system 


$$
\begin{array}{r}
\frac{\partial h}{\partial t}+\nabla \cdot\left(\frac{h^{3}}{3 \mu(\phi)}\left[\gamma \nabla \nabla^{2} h-g_{\perp}\left(\nabla(\rho(\phi) h)-\frac{5}{8} h \nabla \rho(\phi)\right)+\rho(\phi) \mathbf{g}_{\|}\right]\right)=0 \\
\frac{\partial(\phi h)}{\partial t}+\nabla \cdot\left(\frac{\phi h^{3}}{3 \mu(\phi)}\left[\gamma \nabla \nabla^{2} h-g_{\perp}\left(\nabla(\rho(\phi) h)-\frac{5}{8} h \nabla \rho(\phi)\right)+\rho(\phi) \mathbf{g}_{\|}\right]\right. \\
\left.+\phi h(1-\phi) f(\phi) w(h) \mathbf{v}_{\mathbf{s}}\right)=0 .
\end{array}
$$

Next we nondimensionalize the equations for the constant flow rate problem, with the rescaling used in [3] for a clear fluid. If the upstream gate height $h_{0}$ represents a typical film thickness, then the first- and fourth-order terms in (2.18) are comparable at a length scale $x_{0}=\left(\ell^{2} h_{0}\right)^{1 / 3}$, where $\ell=\gamma / \rho_{f} g_{\|}$is the capillary length. The time derivative is on the same scale as well if $t \sim t_{0}=\mu_{f} x_{0} / C a \gamma$, where $C a=h_{0}^{2} / 3 \ell^{2}$ is the dimensionless capillary number. Defining $\tilde{h}=h / h_{0}, \tilde{x}=x / x_{0}, \tilde{t}=t / t_{0}$, $\tilde{\rho}(\phi)=1+\Delta \phi, \tilde{\mu}(\phi)=\left(1-\phi / \phi_{\max }\right)^{-2}, \tilde{w}(\tilde{h})=w(h)$, and dropping the tildes, and replacing $\nabla$ with $\partial / \partial x$ in anticipation of a $y$-independent solution, we obtain the dimensionless system

$$
\begin{array}{r}
\frac{\partial h}{\partial t}+\frac{\partial}{\partial x}\left(\frac{h^{3}}{\mu(\phi)}\left[h_{x x x}-D(\theta)\left((\rho(\phi) h)_{x}-\frac{5}{8} h \rho(\phi)_{x}\right)+\rho(\phi)\right]\right)=0 \\
\frac{\partial(\phi h)}{\partial t}+\frac{\partial}{\partial x}\left(\frac{\phi h^{3}}{\mu(\phi)}\left[h_{x x x}-D(\theta)\left((\rho(\phi) h)_{x}-\frac{5}{8} h \rho(\phi)_{x}\right)+\rho(\phi)\right]+\right. \\
\left.v_{s} \phi h(1-\phi) f(\phi) w(h)\right)=0 .
\end{array}
$$

Here $D(\theta)=(3 C a)^{1 / 3} \cot \theta$ is a parameter measuring the relative importance of the 2nd-order terms.

The above nondimensionalization represents the scales at which all terms in (2.18) are equally significant. However, at length scales $x \gg x_{0}$, the second- and fourth-order terms become small, and can be considered simply a weak diffusive regularization to the dominant first-order system,

$$
\begin{aligned}
\frac{\partial h}{\partial t}+\frac{\partial}{\partial x}\left(h^{3} \rho(\phi) / \mu(\phi)\right) & =0 \\
\frac{\partial(\phi h)}{\partial t}+\frac{\partial}{\partial x}\left(\phi h^{3} \rho(\phi) / \mu(\phi)+v_{s} \phi h(1-\phi) f(\phi) w(h)\right) & =0 .
\end{aligned}
$$

Zhou et al. presented this alternative scaling and presented numerical evidence that the higher-order terms can be neglected. Note also that the second-order terms can also be dropped regardless of the length scale if $\theta=\pi / 2$.

Finally, a discussion is necessary of the microscopic contact-line physics, for which we rely on literature dealing with pure fluids. It has been shown [14] that the no-slip boundary condition we have employed above requires infinite viscous energy dissipation in the vicinity of a moving contact line. This singularity is removed if the fluid-solid boundary condition is modified to allow finite slip [20], which generally takes the form

$$
\left.\mathbf{v}\right|_{z=0}=\left.b \frac{\partial \mathbf{v}}{\partial z}\right|_{z=0}
$$

where $b$ is a length on the order of the molecular size. In fact, the dissipation (and consequently the large scale flow) depends only logarithmically on $b$, so its value need 
not even be known precisely. This slip length has been observed experimentally, and is known to be particularly large (on the order of microns [33]) for polymer liquids such as the PDMS used in [59]. Another technique used to model the contact line derives from attractive Van der Waals forces between the fluid and solid, which for many wetting films (again including PDMS on acrylic) causes a precursor film of microscopic thickness to extend ahead of the apparent contact line [1, 10]. A detailed description of the thermodynamic wetting problem is needed to predict the properties of this precursor film, and while the dissipation in the precursor is large, the dissipation in the macroscopic flow near the contact line is again only weakly dependent on the precursor thickness [13]. Correspondingly, models that simply impose a thickness $b$ for the precursor have been used successfully $[3,55]$, and been seen to give similar predictions to the slip model with the same value of $b[41,51]$.

In this work, we choose the precursor model because it simplifies the analysis by preserving the symmetry of the Riemann problem, discussed in $\S 3$ below. With a precursor of nondimensional thickness $b \ll 1$ and concentration $\phi_{R}$, the initial conditions for the constant flow rate problem are

$$
\left.(h, \phi)\right|_{t=0}=\left\{\begin{array}{lll}
\left(1, \phi_{L}\right) & \text { if } \quad x<0 \\
\left(b, \phi_{R}\right) & \text { if } \quad x>0
\end{array} .\right.
$$

Both $b$ and $\phi_{R}$ are model parameters not determined by the bulk flow, and must be specified. Since the film depths are on the order of millimeters, meaningful values for $b$ are between $10^{-6}$ and $10^{-1}$, corresponding to precursors no thinner than the molecular scale. Reasonable values for the concentration $\phi_{R}$ are between 0 and $\phi_{L}$. We mainly consider $\phi_{R}=\phi_{L}$ for definiteness, but also discuss $\phi_{R}=0$.

The system (2.20) is related to thin film equations that have been studied for pure fluids. If $\phi(x, t) \equiv 0$ or more generally $\phi=\phi_{0} \geq 0$ and $a=0$ (so that $\mathbf{v}_{\mathbf{s}}=0$ ), the system degenerates to the single equation (1.1) studied by Huppert [21]. Huppert finds a fundamental solution for this equation, that is, a solution with Dirac mass initial data, in the form of a combination rarefaction and shock (defined in $\S 3$ )), and finds this solution describes a constant volume experiment he conducted. Bertozzi et al. studied a variant of (1.1) in which Marangoni forcing competes with gravity, resulting in more complex shock structures [4]. Lubrication models have given rise to pairs of coupled equations describing a thin films containing surfactant [22, 35]. Also related are models for sedimenting mixtures [31] in which the particle concentration exhibits kinematic shocks.

3. The Riemann Problem for Systems of Conservation Laws. This section reviews the theory of systems of nonlinear conservation laws in one dimension, of which (2.20) is an example. This class contains equations of the form

$$
\frac{\partial U}{\partial t}+\frac{\partial}{\partial x} F(U)=0, \quad U, F(U) \in \Omega \subset \mathbb{R}^{n} .
$$

Although initial-value problems for (3.1a) are not in general well-posed, there is a large body of analytical techniques for finding and characterizing solutions when they exist [32]. The analysis is especially simplified for the Riemann problem, in which the initial data is a step function

$$
U(x, 0)=\left\{\begin{array}{lll}
U_{L} & \text { if } & x<0 \\
U_{R} & \text { if } & x>0
\end{array},\right.
$$


such as (2.22) with uniform concentration.

Both the equation and initial data of the Riemann problem can be expressed in terms of the single variable $\xi=x / t$, and this symmetry extends to solutions as well. Imposing this form on the solution reduces the problem to finding a heteroclinic orbit for the autonomous system

$$
\begin{gathered}
{[J(U(\xi))-\xi I] \dot{U}(\xi)=0,} \\
U(-\infty)=U_{L}, \quad U(+\infty)=U_{R},
\end{gathered}
$$

where $J(U)$ is the Jacobian derivative of the flux function $F$. Smooth solutions of (3.1a), known as rarefactions, are therefore either constant or vary along integral curves $R_{i}$ of a Jacobian eigenvector $r_{i}$. For this reason, most existence results apply to strictly hyperbolic systems, in which the eigenvalues are real and distinct.

Equation (3.2a) also requires that rarefaction solutions be parametrized by the corresponding eigenvalue $\lambda_{i}$, which is possible only if $\lambda_{i}$ is strictly increasing on $R_{i}$ between $U_{L}$ and $U_{R}$. We discuss here the simplified case when $F$ satisfies the genuine nonlinearity condition, that $\lambda_{i}$ varies strictly monotonically along $R_{i}$ for all $i$ and $R_{i}$, and consider the more general case in the appendix.

In a genuinely nonlinear system $R_{i}(U)$ consists of two connected curves $R_{i}^{+}(U)=$ $\left\{U^{\prime} \in R_{i}(U) \mid \lambda_{i}\left(U^{\prime}\right)>\lambda_{i}(U)\right\}$ and $R_{i}^{-}(U)=\left\{U^{\prime} \in R_{i}(U) \mid \lambda_{i}\left(U^{\prime}\right)<\lambda_{i}(U)\right\}$, and a connecting orbit exists when $U_{L}=U$ and $U_{R} \in R_{i}^{+}(U)$, or $U_{R}=U$ and $U_{L} \in$ $R_{i}^{-}(U)$. Consequently smooth solutions do not exist for general data, and solutions are generally sought from the larger class of weak solutions.

A weak solution to the conservation law (3.1a) is an $L^{\infty}$ function $U(x, t)$ that in addition to the initial condition satisfies

$$
\int_{x_{1}}^{x_{2}}\left(U\left(x, t_{2}\right)-U\left(x, t_{1}\right)\right) d x+\int_{t_{1}}^{t_{2}}\left(F\left(U\left(x_{2}, t\right)\right)-F\left(U\left(x_{1}, t\right)\right)\right)=0
$$

for all $x_{2}>x_{1}$ and $t_{2}>t_{1}>0$. This includes all smooth solutions to (3.1a), but also allows discontinuities along a curve $x=s t$ that satisfies the vector Rankine-Hugoniot condition

$$
F\left(U^{+}\right)-F\left(U^{-}\right)=s\left(U^{+}-U^{-}\right)
$$

where $U^{-}$and $U^{+}$are the values of $U$ on either side of the discontinuity. The Hugoniot locus $H\left(U^{-}\right)$is defined as the set of $U^{+}$that satisfy (3.4) for some $s$. [Note that while the symmetry of (3.4) implies $U_{2} \in H\left(U_{1}\right)$ is equivalent to $U_{1} \in H\left(U_{2}\right)$, it does not follow that $H\left(U_{1}\right)=H\left(U_{2}\right)$ ].

Such weak solutions are not unique, however, and a method must be chosen to select a single solution. Various criteria, known as entropy conditions, have been proposed in order to distinguish the shock, or admissible discontinuity, from any other weak solutions. One condition, the method of viscous profiles, is motivated by the fact that conservation laws often appear physically as approximations to higher-order regularized equations such as

$$
\frac{\partial}{\partial t} U^{\epsilon}+\frac{\partial}{\partial x} F\left(U^{\epsilon}\right)=\epsilon \frac{\partial^{2}}{\partial x^{2}} U^{\epsilon}
$$

which are well-posed for $\epsilon>0$. A solution to (3.1a), according to this method, should be stable in the sense that it appears as the pointwise limit in $x, t$ of solutions $U_{\epsilon}$ 
to (3.5) as $\epsilon \rightarrow 0$. This condition has the advantage of a clearly desirable physical interpretation that assures shock solutions are unique, however it has the drawback of being difficult to verify.

A simpler method from the analytical perspective is the Lax entropy condition, which is equivalent to the viscous profile condition for a certain class of scalar conservation laws. This method relies on strict hyperbolicity to index the eigenvalues $\lambda_{i}$ of $J(U)$ in increasing order for each $U$. These eigenvalues represent the characteristic speeds at which the equation propagates information, as can be seen in rarefaction solutions to the Riemann problem in the persistence of the left state $U_{L}$ for $x \leq \lambda_{i}\left(U_{L}\right) t$ and the right state $U_{R}$ for $x \geq \lambda_{i}\left(U_{R}\right) t$. The Lax entropy condition requires the discontinuity be continually reinforced by conflicting information from a single characteristic field, i.e. it moves with a speed $s$ that satisfies

$$
\lambda_{i}\left(U_{L}\right)>s>\lambda_{i}\left(U_{R}\right)
$$

for exactly one $i$. That characteristic is emphasized by calling the discontinuity an $i$-shock.

In a neighborhood of any $U$ the Hugoniot locus $H(U)$ consists of two smooth curves intersecting at $U$, and the four branches leaving $U$ correspond to the four cases of 1- or 2-shocks with $U$ as the right or left state. We denote the continuations of these branches by $U_{i}^{+}$if $U$ is the left state and $U_{i}^{-}$if $U$ is the right state. The allowable connections $C_{i}^{+}\left(U_{L}\right)=R_{i}^{+}\left(U_{L}\right) \cup S_{i}^{+}\left(U_{L}\right)$ through the $i$-th characteristic also locally form a smooth curve for each $i$. The variation of an $i$-shock or $i$-rarefaction solution is confined to the interval $\left\{\xi: \min \left(\lambda_{i}\left(U_{L}\right), \lambda_{i}\left(U_{R}\right)\right)<\xi<\max \left(\lambda_{i}\left(U_{L}\right), \lambda_{i}\left(U_{R}\right)\right)\right\}$, so compound connections can be generated by stringing together waves of different characteristics as long as $\xi$ increases with $i$. In fact, $\left\{C_{i}^{+}\right\}_{i=1}^{n}$ locally generate a smooth coordinate system, so if $U_{R}$ is sufficiently close to $U_{L}$ the Riemann problem is well-posed.

Existence of solutions for large data depends on the topology of $H(U)$. A famous example of a system with no solutions for certain Riemann data is the Keyfitz-Kranzer equation (5.1) [29], in which $H(U)$ is compact. A bounded Hugoniot locus implies a bound on the strength of a shock, and consequently some large-data Riemann problems have no weak solutions. Section 5 describes a theory for such systems relating the regularized profiles to a Dirac mass, however this theory is far from complete.

A final complication to the selection of weak solutions is the nature of the regularization actually present in the physical system. The Lax and Oleinik conditions are intended to admit those shocks that appear as viscous limits under the simplest possible regularization. If the actual regularization is different, the viscous profiles could converge to a weak solution other than that selected by the entropy criteria. This possibility is indeed relevant to conservation laws describing thin films, which are generally regularized by nonlinear fourth-order capillary terms such as in (2.19). In fact, a scalar thin film equation with similar regularization is known to select an entropy-violating double-shock solution, rather than the single-shock entropy solution [4].

4. Particular Solutions. The system (2.20) is physically meaningful for $(h, \phi)$ in the phase space $\Omega=\left\{(h, \phi): 0<h, 0<\phi<\phi_{\max }\right\}$, which can also be expressed in terms of the conserved quantities $u \equiv h$ and $v \equiv \phi h$ as $\left\{(u, v) \in \mathbb{R}^{2}: 0 \leqslant v<\phi_{\max } u\right\}$. While the above theory depends on the latter parameterization, the flux functions are most simply expressed in terms of $h$ and $\phi$, so we retain these variables for presenting our results. 


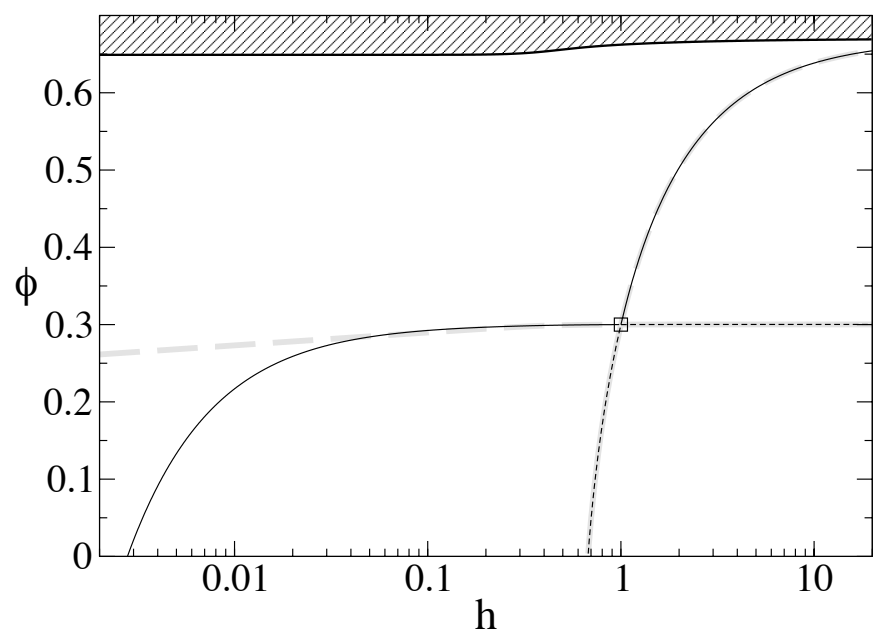

FIG. 4.1. The phase space of the reduced model, and the connections from $\left(h_{0}, \phi_{0}\right)=(1.0,0.3)$, $\square$. The system is hyperbolic except in the shaded region. Black lines represent shock connections and gray represents rarefactions. Solid lines are connections to the right, i.e. the $\left(h_{0}, \phi_{0}\right)$ is the left state, and dashed lines are connections to the left. 1-waves and 2-waves can be distinguished by their slope at $\left(h_{0}, \phi_{0}\right)$ : 2-waves are nearly horizontal at this scale.

The simple connections for a left state of $\left(h_{L}, \phi_{L}\right)=(1.0,0.3)$ are shown in figure 4.1. The rarefaction curves have been integrated from (3.2a) by a RungeKutta method, and $H\left(U_{L}\right)$ has been calculated by eliminating $s$ from (3.4) at each point and solving the resulting equations for $u$ and $v$. For a given shock connection, the shock speed can be recovered by substituting $u$ and $v$ back into (3.4).

Since $h$ was rescaled by the film thickness set at the upstream gate, we choose $(h, \phi)=(1.0,0.3)$ as the left state. For a specified right state $\left(b, \phi_{R}\right)$, representing the precursor film, a solution to the Riemann problem can be determined by finding intersections between the two connection diagrams. In figure 4.2 we have plotted the possible shock-shock connections for four values of $b$ with $\phi_{R}=\phi_{L}$. At $b=0.1$ there is a solution with a 1-shock from the upstream state to an intermediate height and concentration slightly larger, and a 2-shock from this intermediate state to the precursor. As the precursor becomes thinner, the height and concentration of this intermediate state increase. For $b=0.01$ the intermediate state is approximately $(h, \phi)=(1.1757,0.3663)$, and in figure 4.3 we compare this connection with a numerical solution with the same initial data, and find both shock speeds and the height and concentration of the ridge are in agreement. The numerical solution was calculated using the Lax-Freidrichs finite difference method with grid spacing $3.3 \times 10^{-7}$ and timestep $3.3 \times 10^{-7}$.

At $b=0.008$ the Hugoniot locus has undergone a bifurcation such that the 1and 2-shock curves are no longer distinct, and an additional connected component has appeared. The shock speed and characteristic speeds coincide at several points along these curves, such that various sections correspond to 1-shocks, 2-shocks, or are not admissible at all. There is still a shock-shock connection that satisfies the Lax 


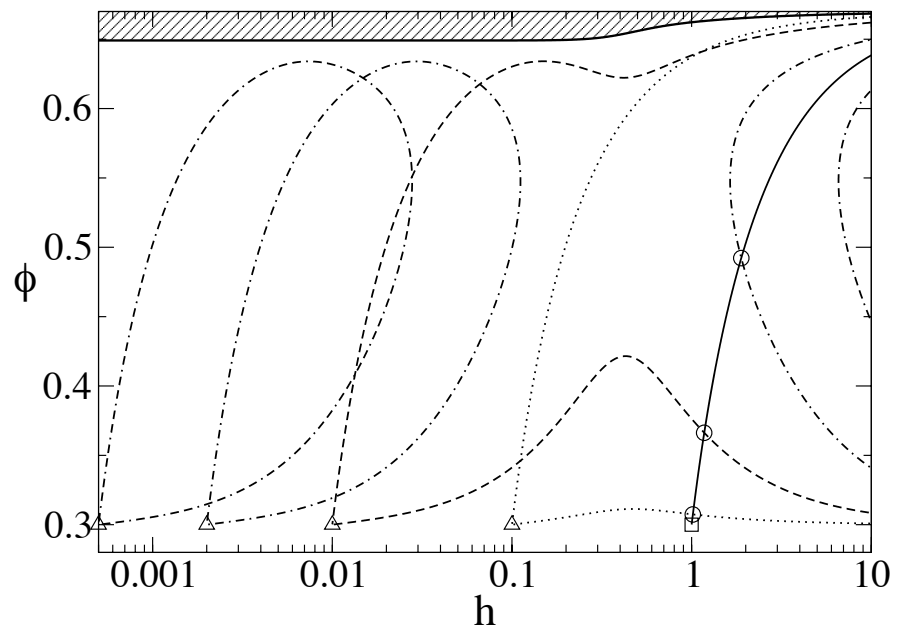

FIG. 4.2. 1-shock connections (solid line) from an upstream state $\left(h_{L}, \phi_{L}\right)=(1.0,0.3)(\square)$ and 1- and 2-shock connections from four precursor states $\left(h_{R}, \phi_{R}\right)=(b, 0.3)(\triangle)$ where $b=0.1$ (dot), 0.01 (dash), 0.002 (dot-dash), and 0.0005 (dot-dash-dash). The solutions involve an intermediate state between the two shocks, marked by $\bigcirc$. As b becomes small, the Hugoniot locus undergoes a bifurcation, becoming disconnected, and ultimately fails to produce a shock solution.

entropy condition. At $b=0.0015$, however, there are no longer any intersections, and since the rarefactions also fail to intersect, this initial data has no solution. We will discuss this case further in $\S 5$.

As shown in figure 4.1, the equations using (2.13) are neither strictly hyperbolic nor genuinely nonlinear on this entire domain. Hyperbolicity fails near the maximum concentration, as the eigenvalues become complex and the equations become elliptic. It is not clear whether this feature is desired in a model of the thin film. Change of type certainly complicates the mathematical question of well-posedness for such a system, but remembering that the first-order system is only an approximation to the full fourth-order model, this may be an inconvenience rather than a physical flaw. Physical models proposed for dry granular materials result variously in hyperbolic, parabolic, and elliptic equations, so physically the change of type does not seem altogether unreasonable.

If the concentration in the precursor is taken to be 0 rather than $\phi_{L}$, double-shock solutions again occur and the same non-existence issue occurs for small $b$. For larger $b$, an additional type of solution occurs consisting of a 1-rarefaction and a 2-shock, with both $h$ and $\phi$ in the intermediate state less then their values at the left. A numerical solution for this case is shown in figure 4.4, again computed using the Lax-Freidrichs finite difference method in a moving frame. This behavior does not correspond to anything observed in the experiments of Zhou et al., and is probably observable only for heavily pre-wet substrates if at all.

5. Singular Shocks. The problem of non-existence due to non-trivial Hugoniot topology has been studied before, and a weaker form of solution known as a singular shock has been described. An illustrative example is the Keyfitz-Kranzer equation 


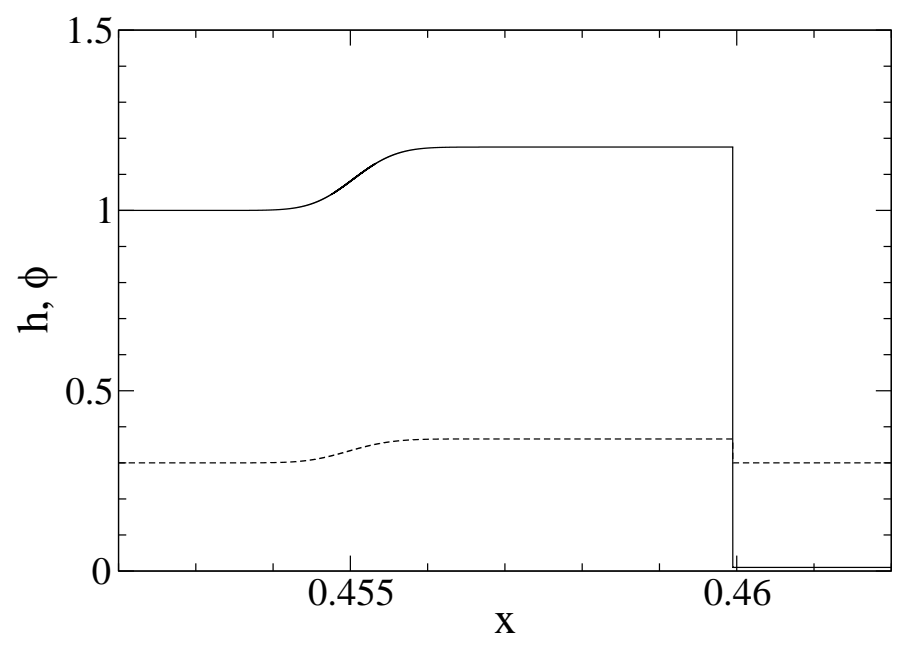

FIG. 4.3. Film thickness (solid) and concentration (dashed) of a numerical solution of the conservation laws at $t=1$, with $\left(h_{L}, \phi_{L}\right)=(1.0,0.3)$ and $\left(h_{R}, \phi_{R}\right)=(0.01,0.3)$. Numerical diffusivity generally affects the leading shock less than the trailing shock, since for the latter one of the characteristic speeds is close to the shock speed.

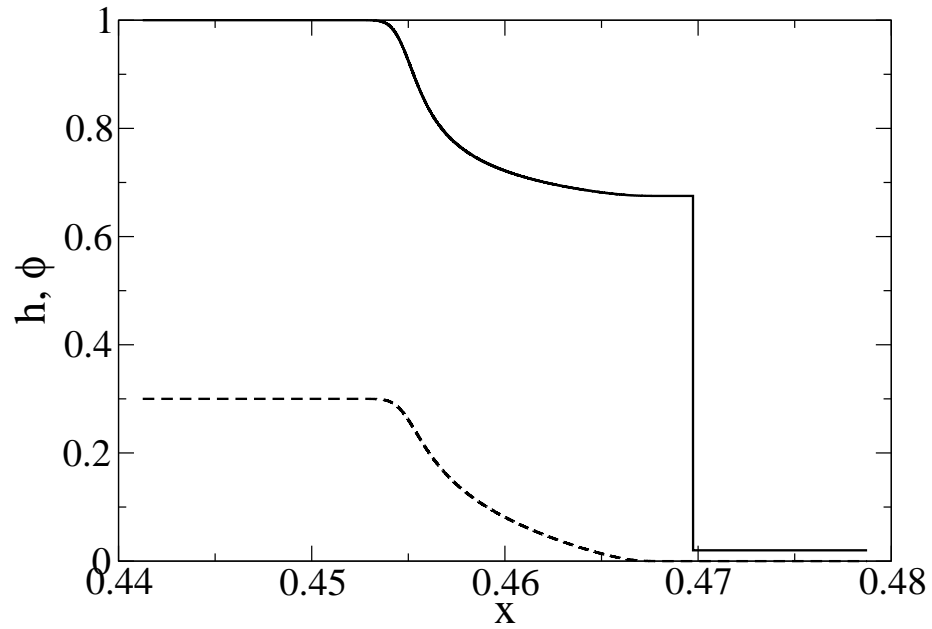

FIG. 4.4. Numerical solution of the conservation laws at $t=1$, with $\left(h_{L}, \phi_{L}\right)=(1.0,0.3)$ and $\left(h_{R}, \phi_{R}\right)=(0.02,0)$, corresponding to a 1-rarefaction and 2-shock. While some of the smoothness is due to numerical diffusivity, the 1-rarefaction can also be distinguished from a 1-shock by the fact that both $h$ and $\phi$ are less than their values on the left. 
$[29]$

$$
\frac{\partial}{\partial t}\left(\begin{array}{c}
u \\
v
\end{array}\right)+\frac{\partial}{\partial x}\left(\begin{array}{c}
u^{2}-v \\
\frac{1}{3} u^{3}-u
\end{array}\right)=0
$$

which is everywhere both strictly hyperbolic and genuinely nonlinear, but for all $U=(u, v)$ the Hugoniot locus is compact, specifically figure-8 shaped. Thus shocks can only connect states that are sufficiently close, and certain Riemann problems have no classical solution.

In [27], Keyfitz and Kranzer present three sequences of functions $U^{\epsilon}(\xi=x / t)$ to (5.1) that approximately solve (5.1) as $\epsilon \rightarrow 0$ but are also singular in this limit. The first sequence results from an asymptotic expansion of the solution to the regularized equation

$$
\frac{\partial U}{\partial t}+\frac{\partial}{\partial x} F(U)=\epsilon t \frac{\partial^{2} U}{\partial x^{2}}
$$

in $\epsilon$, and the second and third are explicitly constructed from $C^{\infty}$ functions and piecewise constant functions. They introduce a space of measures in which these sequences converge to a limit involving Dirac-like masses superimposed on a classical shock. They also propose overcompression as an admissibility requirement for singular shocks, i.e. (3.6) must hold for both characteristics; if singular shocks are accepted under this restriction (5.1) is well posed for all Riemann data. However, these conclusions are restricted to (5.1). Also, Keyfitz and Kranzer emphasize that while the limiting measures appear as limits of approximate solutions, no well-defined criterion has been proposed by which the limits themselves can be called solutions.

Sever discusses the selection mechanism for singular shocks in a more general context in [48]. For a distribution solution

$$
U(x, t)=M(t) \delta(x-s t)+ \begin{cases}U_{L} & \text { if } x<s t \\ U_{R} & \text { if } x>s t\end{cases}
$$

characterized by a point mass $M(t)$ located at $x=s t$, conservation implies the singular mass must satisfy

$$
\frac{d M}{d t}=s\left(U_{R}-U_{L}\right)-\left[F\left(U_{R}\right)-F\left(U_{L}\right)\right] .
$$

Since the speed $s$ is unknown, this is an undetermined system for the $n+1$ parameters $d M / d t$, s. For (5.1), Keyfitz and Kranzer determined unique solutions by requiring the first component of $M$ to vanish, justified by an argument specific to that system. Sever writes that this last constraint generally comes from properties of the system such as symmetry groups or a convex entropy function. The proper constraint for system (2.20) is not yet apparent.

Equations (2.20) with regularization (3.5) also show behavior consistent with a singular shock. In order to investigate this, numerical solutions were generated with a fully implicit centered difference scheme on a moving nonuniform grid. The number of grid points at each mesh size was fixed, however every 10 time steps the grids were rearranged using cubic interpolation as necessary to center the area of maximum resolution around the singularity. Meanwhile the entire computational domain moved a constant speed chosen to approximately match the speed of the discontinuity. The scaling of the regularized solution satisfies $U^{\epsilon}(x, t)=U^{1}(\epsilon x, \epsilon t)$, so rather than take $\epsilon \rightarrow 0$ we fixed $\epsilon=1$ and evaluated the solution at long times. 
Figure 5.1 contains the results of this calculation. Both components of the singular mass increase linearly in time, as required by (5.4), and the singularity is overcompressive. As the singularity evolves in time the maximum height and concentration grow, and at $t \approx 3 \times 10^{8}$ the concentration exceeds the packing fraction. While this linear second-order diffusion may behave differently from the nonlinear fourth-order diffusion in (2.19), the unphysical concentrations suggest the model may be inaccurate for high concentrations.
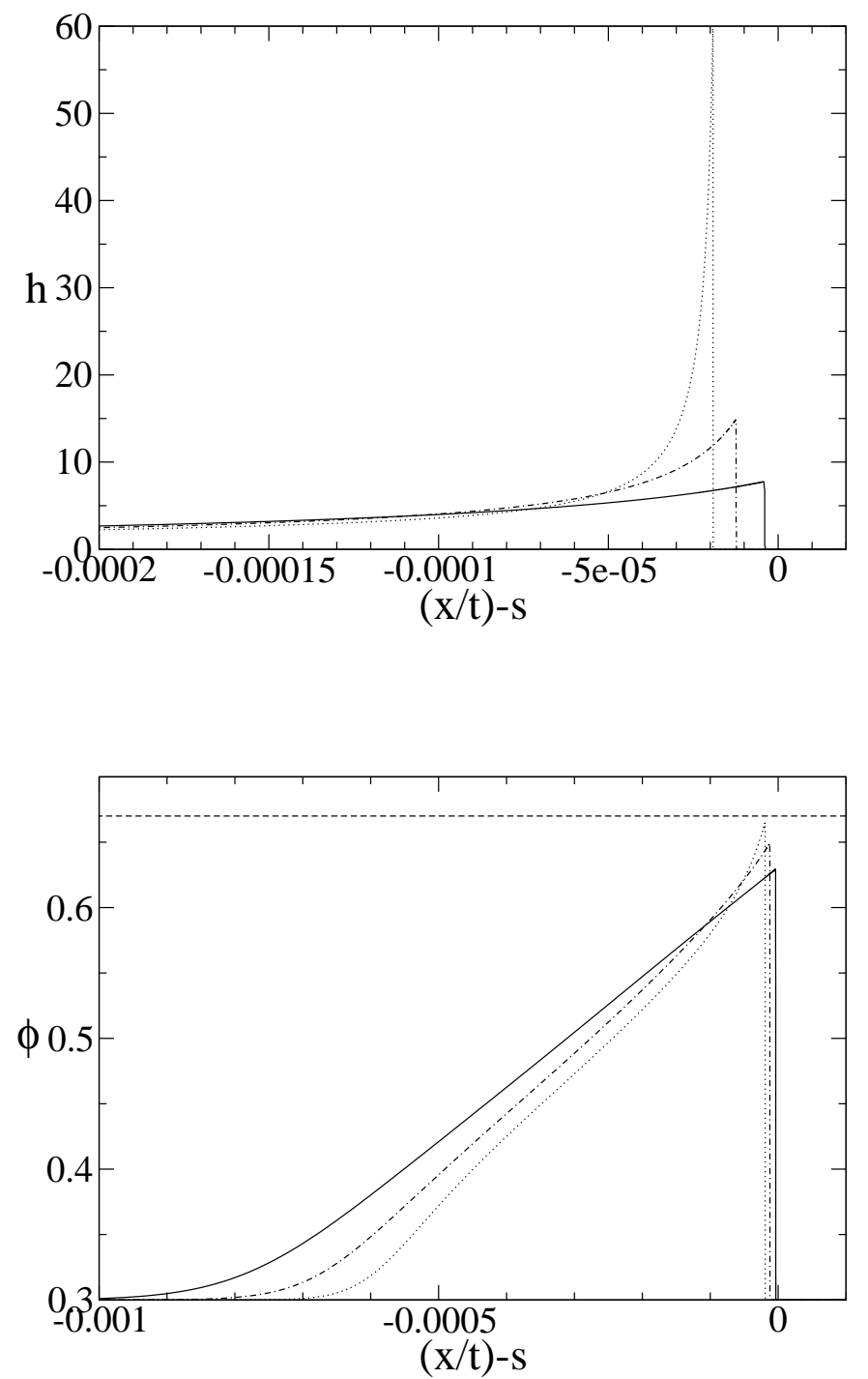

FIG. 5.1. Film thickness (top) and particle concentration (bottom), from numerical solutions of the regularized system (3.5) in the singular shock regime, with $b=0.001, \phi_{0}=0.3$, and $\epsilon=1$, calculated on a grid moving at speed $s=0.45547$ and evaluated at times $5 \times 10^{7}$ (solid), $1 \times 10^{8}$ (dot-dash), and $2 \times 10^{8}$ (dot). The dashed line on the bottom plot marks $\phi_{m}$. 
6. Alternative Settling Function. In this section we propose a modification to the unregularized system (2.20) to ensure the concentration does not exceed $\phi_{\max }$. We begin with a heuristic explanation of how (2.13) may be incompatible with (2.1) in the limit $\phi \rightarrow \phi_{\max }$. The volume-averaged velocity is controlled by $\mu(\phi)^{-1}$, which vanishes in this limit, while $f_{R Z}(\phi)$ and hence the relative flux is nonzero. This imposes a forward flux of particles with no net volume flux, requiring fluid therefore to move backward. This situation is probably unrealistic, because the limit $\mu(\phi) \rightarrow \infty$ is intended to model the case when the particles are packed tightly enough to prevent any shear flow. In that case, it seems more appropriate to model the particles as an immobile porous medium, with a Darcy's law flux of pure fluid and $\mathbf{v}_{\text {rel }}<0$. Incorporating such a transition into the current model presents challenges, as the particle velocity must be specified relative to the laboratory frame rather than the fluid, essentially changing to a two-fluid model at high concentrations. A much simpler alternative is to simply let $\mathbf{v}_{\text {rel }}$ vanish along with $\mathbf{v}$ at $\phi=\phi_{\max }$; this is readily accomplished by using the hindered settling function proposed by Buscall et al. [7]

$$
f_{B}(\phi)=\left(1-\phi / \phi_{\max }\right)^{5}
$$

instead of (2.13). The two settling functions are plotted in figure 6.1.

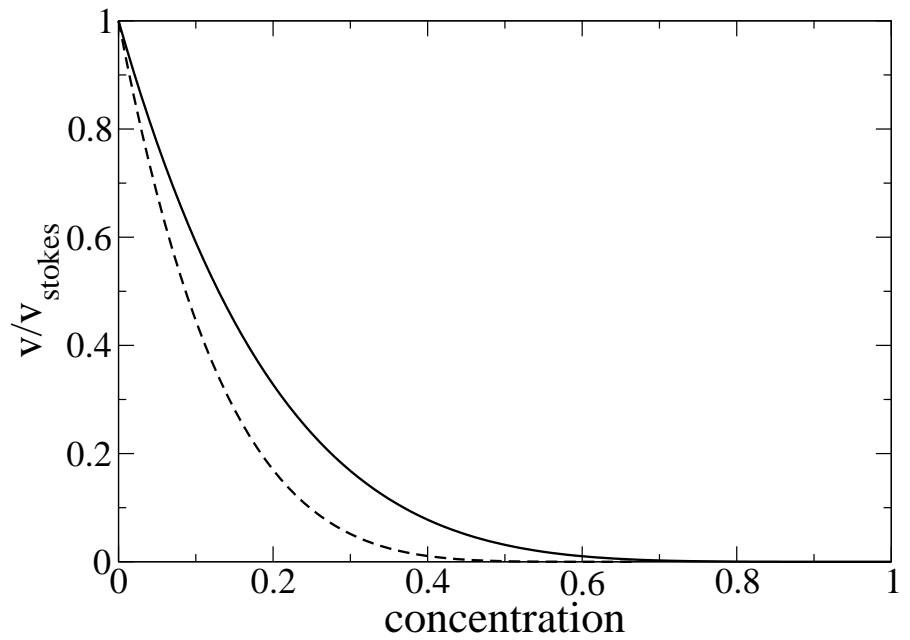

FIG. 6.1. Two forms of the hindered settling function. The Richardson-Zaki form (solid line) vanishes at concentration 1.0, another form due to Buscall et al. (dashed line) vanishes at the packing fraction 0.67 .

With this modification, solving the Riemann problem is simplified in two significant ways: the equations are strictly hyperbolic throughout the relevant domain $\Omega$, and the bifurcation causing shock solutions to break down does not occur. In figure 6.2 we have plotted shock-shock connections for four values of $b$. These solutions exist even for very small precursors, so the system appears to be well-posed regardless of $b$. Figure 6.3 summarizes the manner in which the type of solution depends on the settling function and the Riemann data. 


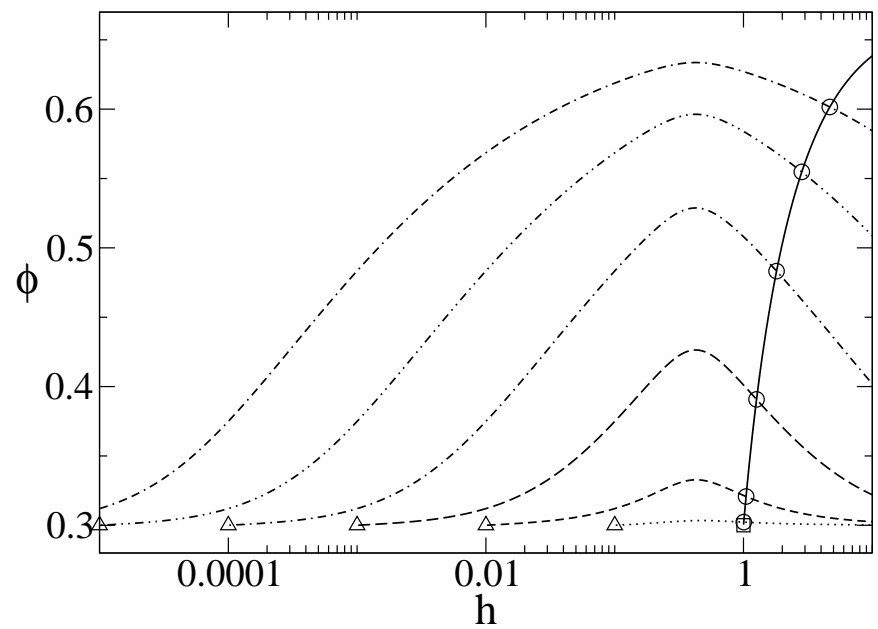

FIG. 6.2. Shock connections using the settling function $f_{B}(\phi)=\left(1-\phi / \phi_{\max }\right)^{5}$ instead of $f_{R Z}(\phi)$. The bifurcation that caused some initial data to have no solution no longer occurs. The solid line is the 1-shock connection from $\left(h_{L}, \phi_{L}\right),(\square)$, and the 2-shocks are plotted from various precursors $(\triangle)$ given by $b=10^{-1}$ (dot), $10^{-2}$ (short dash), $10^{-3}$ (long dash), $10^{-4}$ (dot-dash), $10^{-5}$ (dot-dot-dash), and $10^{-6}$ (dot-dash-dash). Each solution involves an intermediate state marked by ○.
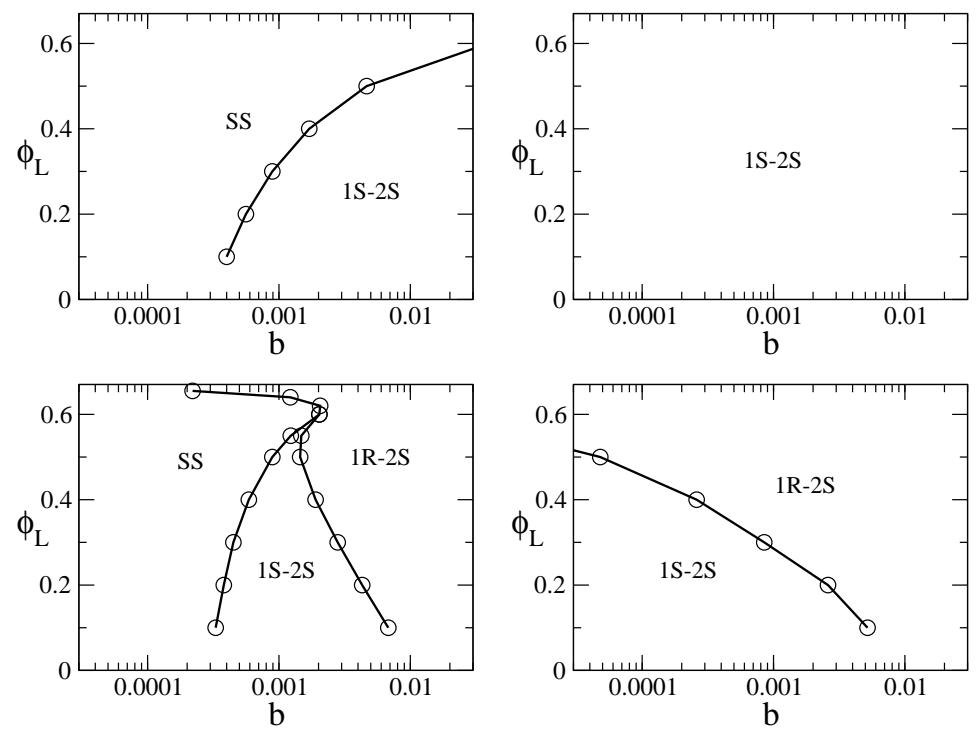

FIG. 6.3. Type of solution (1-rarefaction and 2-shock, 1-shock and 2-shock, or singular shock) as determined by $b$ and $\phi_{L}$ (assuming $h_{L}=1$ and either $\phi_{R}=\phi_{L}$ or $\phi_{R}=0$ ), for both hindered settling functions. Richardson-Zaki settling and $\phi_{R}=\phi_{L}$ (upper left), Richardson-Zaki settling and $\phi_{R}=0$ (lower left), Buscall et al. settling and $\phi_{R}=\phi_{L}$ (upper right), Buscall et al. settling and $\phi_{R}=0$ (lower right). 
In figures 6.4-6.5, we compare the shock solutions to the two systems and their dependence on the precursor $b$. The behavior of the Hugoniot curves in the $f_{R Z}(\phi)$ system, shown in figure 4.2 , implies the intermediate height and concentration approach a maximum value at a critical precursor thickness $b=b_{*} \approx 9 \times 10^{-4}$, below which there is no meaningful solution. As $b \rightarrow 0$ in the $f_{B}(\phi)$ system, the intermediate height increases apparently without bound and the concentration approaches $\phi_{\max }$. We also observed in both limits that the speeds of the 1- and 2- shocks to become approximately equal.

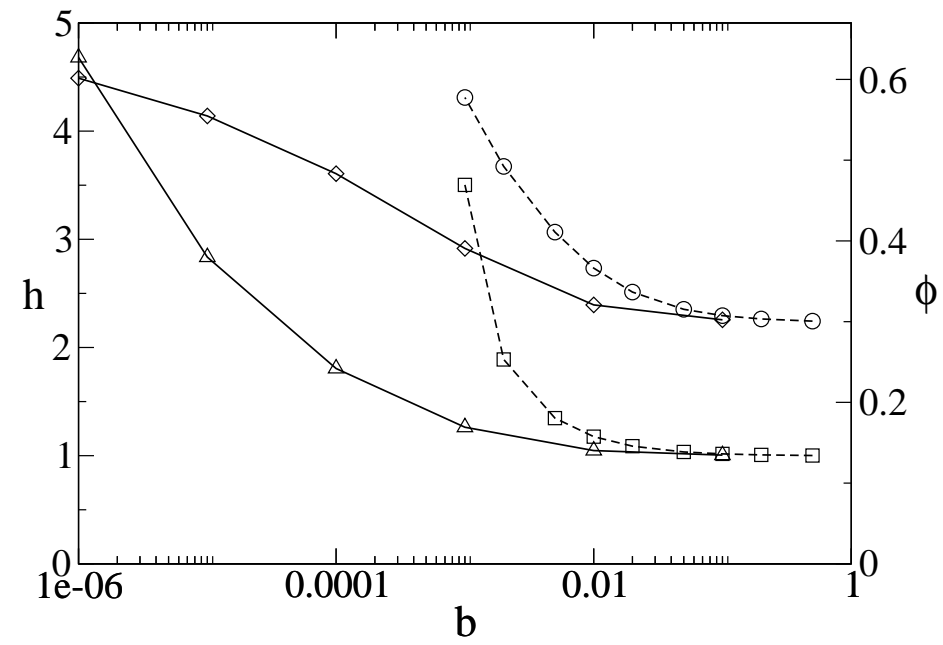

FIG. 6.4. Height and concentration of the intermediate state vs. the precursor thickness $b$. Squares and circles are the height and concentration of solutions using the hindered settling function $f_{R Z}(\phi)$, triangles and diamonds are the height and concentration of solutions using $f_{B}(\phi)$.

7. Conclusion. In $\S 2$, we derived a lubrication model for particle-laden films in the case where particle settling occurs only in the direction of flow. We expect, since variations in the film thickness occur only near the contact line, that the large-scale flow is determined by the first-order terms, which take the form of a hyperbolic pair of conservation laws. When the precursor thickness $b$ is large enough, this system has a double shock solution in qualitative agreement with the experimentally observed particle-rich ridge. For smaller $b$, however, the system has no classical solution. We have confirmed the effect of converging shock speeds that Zhou et al. reported, and find that the speeds appear to become equal at the same value of $b$ for which the classical shock solution breaks down.

At precursor thicknesses for which classical solutions do not exist, we have investigated a simple regularization of the equations for which the solution resembles a singular shock. The height appears unbounded in this solution as $t \rightarrow \infty$, corresponding to weak regularization, and a growing delta mass is concentrated at the shock location. Eventually, the close packing concentration $\phi_{\max }$ is exceeded, and the solution loses physical significance.

A heuristic explanation was offered in $\S 6$ for this behavior in terms of limiting 


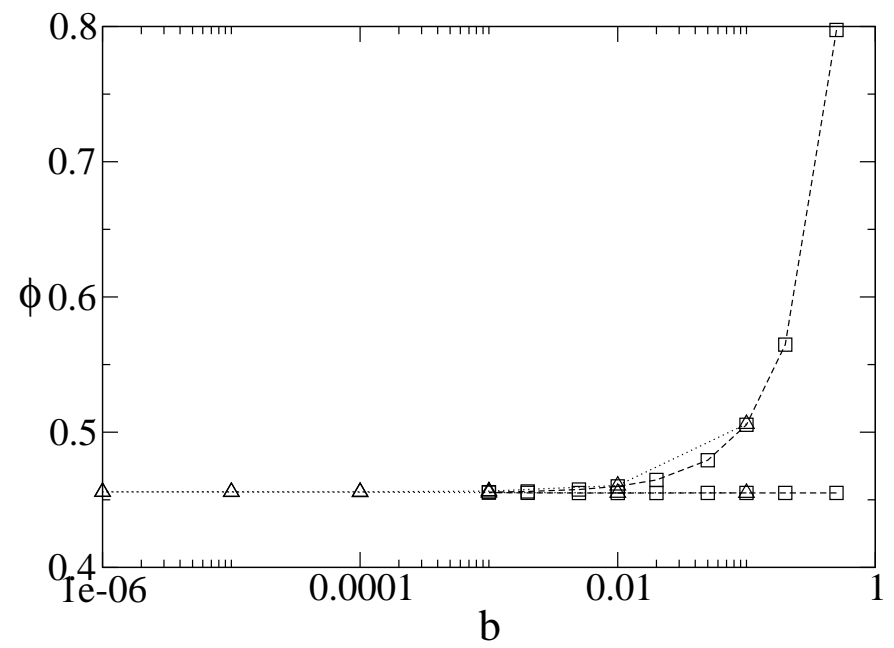

FIG. 6.5. The speed of the shocks that make up the solutions to the connection problem for various precursors. Squares are solutions using the hindered settling function $f_{R Z}(\phi)$, and triangles with $f_{B}(\phi)$.

fluxes as $\phi \rightarrow \phi_{\max }$, suggesting that the relative velocity should also vanish in this limit. This can be achieved by substituting the hindered settling function (6) of Buscall et al. for that of Richardson and Zaki, and the corresponding Riemann problem appears to be well-posed for all precursor thicknesses. We therefore believe the function $f_{B}(\phi)$ is more appropriate for high-concentration flows.

Many interesting questions remain unanswered regarding this model. More work is needed to determine how well the present results concerning the first-order system (2.20) approximate the full fourth-order system (2.19). Also of interest is the stability of the two-dimensional model (2.18) with respect to fingering patterns, as the experiments of Zhou et al. found instability to be suppressed when a particle-rich ridge develops [59]. Other questions arise from the limitations of the current model. Explaining the three distinct settling behaviors observed by Zhou et al. requires a more general model considering particle settling in the normal direction, perhaps balanced by a shear-induced particle flux as in [47]. In addition to explaining the phase diagram, such a model could also help determine whether the assumption in the current model, that particle concentration is constant across the film depth, is reasonable. Changes to the model may also be needed to describe very high concentrations, as suggested in $\S 6$, because contact forces between particles can be expected to become important.

Acknowledgments. The authors would like to thank Professors Barbara Keyfitz and Michael Shearer for insightful comments and suggestions. 
Appendix. Genuine Nonlinearity. While most physical systems are strictly hyperbolic, systems arising naturally are often not genuinely nonlinear. In the Euler equations of compressible flow, one characteristic field is linearly degenerate: $r_{i} \cdot \nabla \lambda_{i} \equiv$ 0 . For this characteristic, $R_{i}(U)$ and $S_{i}(U)$ coincide and connections take the form of contact discontinuities, which satisfy (3.4) with the inequalities in (3.6) replaced by equality. More generally, when the variation of $\lambda_{i}$ along $R_{i}$ changes sign, the strict inequality in (3.6) becomes too restrictive and an entropy condition is needed to select which contact discontinuities are admissible solutions.

For a scalar conservation law, genuine nonlinearity is simply the strict convexity (or concavity) of the flux function $F$. If the function changes concavity, contact discontinuities are chosen by the Oleinik condition [43], that the shock speed $s\left(U_{L}, U_{R}\right)$ satisfies

$$
s\left(U_{L}, U_{R}\right) \leq s\left(U_{L}, U\right)
$$

for any $U$ between $U_{L}$ and $U_{R}$. Liu has provided a generalization to $2 \times 2$ [36] and $n \times n$ systems [37] that requires (A.1) hold for all $U \in H\left(U_{L}\right)$ between $U_{L}$ and $U_{R}$. Both Liu's and Oleinik's conditions reduce to (3.6) for a genuinely nonlinear system. While potentially only a bounded segment of $H\left(U_{L}\right)$ could be available for discontinuous waves, relaxing condition (3.6) provides more solutions by allowing both continuous and discontinuous waves in the same characteristic. Liu provides an existence proof, by constructing such a compound wave. This connection involves a shock to the first point $U_{*}$ satisfying $s\left(U_{L}, U_{*}\right)=\lambda_{i}\left(U_{*}\right)$, followed by a rarefaction from $U_{*}$ to $U_{R} \in R_{i}^{+}\left(U_{*}\right)$. The point $U_{*}$ is both the first local minimum of $s$ along $H\left(U_{L}\right)$, hence the last point for which Liu's entropy condition is satisfied, and the first point for which $\lambda_{i} \geq s$, necessary for a continuing rarefaction wave.

In $(2.20), r_{1} \cdot \nabla \lambda_{1}=0$ holds along the curve shown in figure A.1. For $\left(h_{L}, \phi_{L}\right)=$ $(1,0.3)$ the branches $S_{1}^{+}$and $R_{1}^{-}$nearly coincide, so this branch represents to good approximation the states accessible through a 1-shock, 1-rarefaction compound wave as well. In figure A.2 the eigenvalue and shock speed are plotted on this curve as a function of $\phi$. For $\phi<\phi_{L}$, both speeds increase away from $U_{L}$, indicating a simple rarefaction. With $\phi_{L}<\phi_{*} \approx 0.369$, the shock speed is strictly decreasing with $\phi$ so the connection is a shock satisfying the Liu-Oleinik condition. This case includes the solutions described in $\S 4$ for $b=0.1$ and $b=0.01$. For $\phi>\phi_{*}$ neither simple wave is feasible, but a contact discontinuity from $\phi_{L}$ to $\phi_{*}$ can connect with a rarefaction from $\phi_{*}$ to $\phi$ because $\lambda_{1}$ is now both increasing and greater than the shock speed.

This compound wave is in practice difficult to distinguish from a simple shock. As noted above, the states accessible to a compound wave are nearly the same states lying on $R_{1}$ or $S_{1}$, so the constant state $U_{I}$ appearing between 1-waves and 2-waves cannot easily be used to identify the compound wave. Additionally, figure A.2 demonstrates that $\lambda_{1}$ changes very slowly along its characteristic at intermediate concentrations, so for instance in the presence of numerical diffusion, the rarefaction appears indistinguishable from a shock. Thus although some solutions are necessarily compound waves, their observable properties (other than failing to satisfy the Lax condition) are similar to those of a simple shock. 


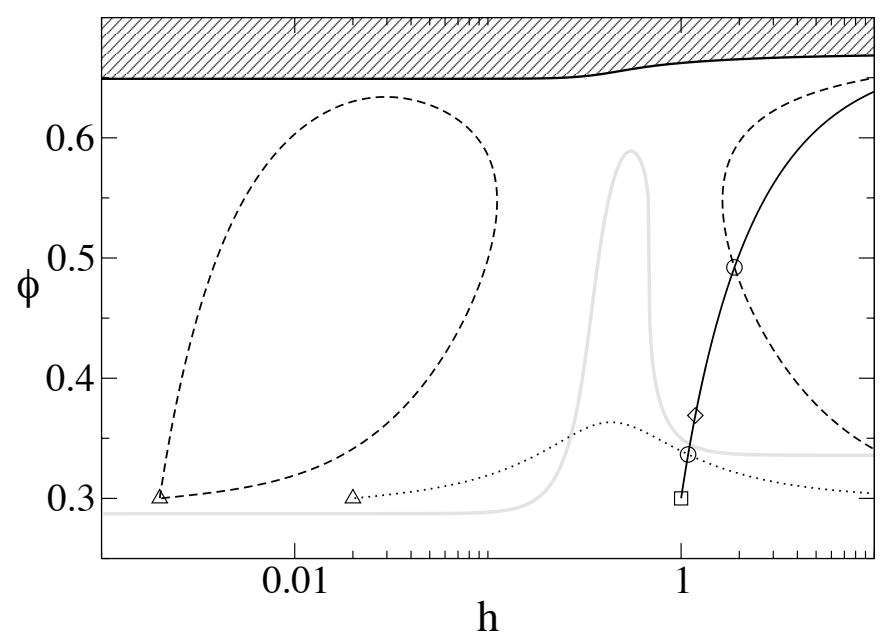

FIG. A.1. Failure of genuine nonlinearity for (2.20): $\nabla \lambda_{1} \cdot r_{1}=0$ on the gray line. Connections from $\left(h_{L}, \phi_{L}\right)=(1.0,0.3)(\square)$ are plotted on the dashed line, which include shocks up to $\left(h_{*}, \phi_{*}\right) \approx$ $(1.18,0.369)(\diamond)$ or a compound shock to $\left(h_{*}, \phi_{*}\right)$ followed by a rarefaction. 2-shocks are plotted from right states $(\triangle)$ for one case $(b=0.02$, dotted line) with a simple 1-shock, 2-shock solution, and another case ( $b=0.002$, dashed line) with a compound 1-shock, 1-rarefaction wave and a 2-shock. The equations are elliptic in the shaded region.

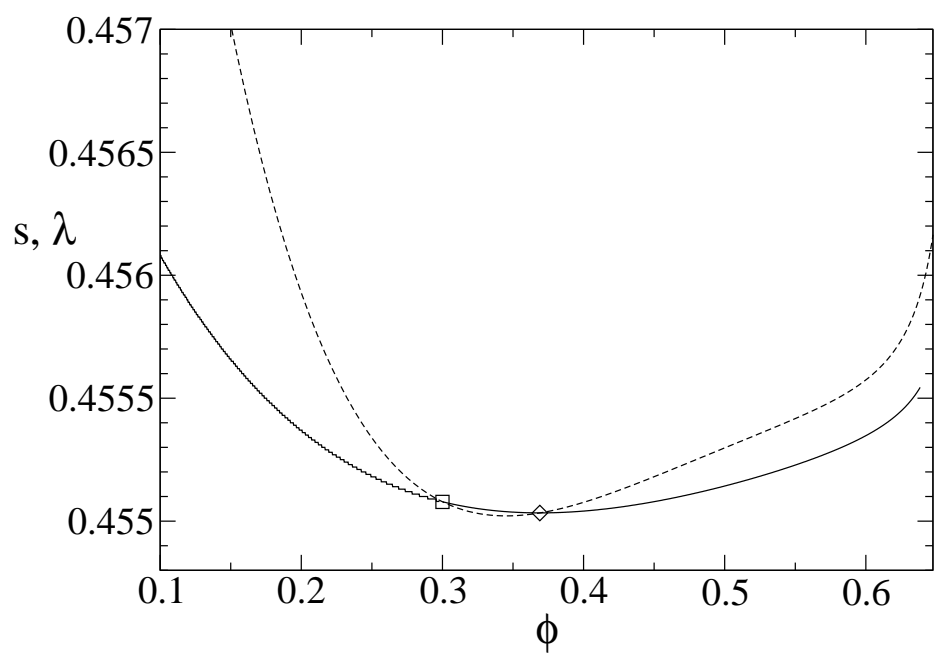

FIG. A.2. Rarefaction speeds (dashed line) and shock speeds (solid) for the connections along the first characteristic from a left state $\left(h_{L}, \phi_{L}\right)=(1.0,0.3),(\square)$, (corresponding to figure 4.1), plotted as a function of the concentration $\phi_{R}$ at the right state. The linear degeneracy curve in figure A.1 indicates the location of the minimum characteristic speed. If $\phi_{R}>\phi_{*} \approx 0.37(\diamond), a$ single shock solution is not admissible and the solution consists of a hybrid shock-rarefaction wave. 


\section{REFERENCES}

[1] D. Ausserré, A. M. Picard, and L. LÉger, Existence and role of the precursor film in the spreading of polymer liquids, Phys. Rev. Lett., 57 (1986), pp. 2671-2674.

[2] R. Benzi, L. Biferale, M. Sbragaglia, S. Succi, and F. Toschi, Mesoscopic modeling of a two-phase flow in the presence of boundaries: The contact angle, Phys. Rev. E, 74 (2006), p. 021509.

[3] A. L. Bertozzi And M. P. Brenner, Linear stability and transient growth in driven contact lines, Phys. Fluids, 9 (1997).

[4] A. L. Bertozzi, A. Münch, And M. Shearer, Undercompressive shocks in thin film flows, Phys. D, 134 (1999), pp. 431-464.

[5] M. P. Brenner And P. J. Mucha, That sinking feeling, Nature, 409 (2001), pp. 568-570.

[6] J. P. Burelbach, S. G. Bankoff, and S. H. Davis, Nonlinear stability of evaporating/condensing liquid films, J. Fluid Mech., 195 (1988), pp. 463-494.

[7] R. Buscall, J. W. Goodwin, R. H. Ottewill, and T. F. Tadros, The settling of particles through Newtonian and non-Newtonian media, J. Colloid Interface Sci., 85 (1982), pp. 7886.

[8] R. E. Caflisch AND J. H. C. LuKe, Variance in the sedimentation speed of a suspension, Phys. Fluids, 28 (1985).

[9] I. C. Carpen and J. F. Brady, Gravitational instability in suspension flow, J. Fluid Mech., 472 (2002), pp. 201-210.

[10] A. M. Cazabat, Wetting films, Adv. Colloid Interface Sci., 34 (1991), pp. 72-88.

[11] R. H. Davis AND A. ACRIVos, Sedimentation of noncolloidal particles at low Reynolds numbers, Annu. Rev. Fluid Mech., 17 (1985).

[12] J. R. DE BRUYN, Growth of fingers at a driven three-phase contact line, Phys. Rev. A, 46 (1992), pp. R4500-R4503.

[13] P. G. De Gennes, Wetting: Statics and dynamics, Reviews of Modern Physics, 57 (1985).

[14] E. B. Dussan V. And S. H. Davis, On the motion of fluid-fluid interface along a solid surface, J. Fluid Mech., 65 (1974), pp. 71-95.

[15] Z. W. Fang, A. A. Mammoli, J. F. Brady, M. S. Ingber, L. A. Mondy, and A. L. GraHAM, Flow-aligned tensor models for suspension flows, Int. J. Multiphase Flow, 28 (2002), pp. 137-166.

[16] R. Goodwin And G. M. Homsy, Viscous flow down a slope in the vicinity of a contact line, Phys. Fluids A, 3 (1991), pp. 515-528.

[17] H. P. GReEnspan, On the motion of a small viscous droplet that wets a surface, J. Fluid Mech., 84 (1978), pp. 125-143.

[18] J. Happel AND H. BRENNER, Low Reynolds number hydrodynamics, with special applications to particulate media, Prentice-Hall, 1965.

[19] L. M. Hocking, Spreading and instability of a viscous fluid sheet, J. Fluid Mech., 211 (1990), pp. 373-392.

[20] C. Huh And L. E. ScRIVen, Hydrodynamic model of steady movement of a solid/liquid/fluid contact line, J. Colloid Interface Sci., 35 (1971), pp. 85-101.

[21] H. E. Huppert, Flow and instability of a viscous current down a slope, Nature, 300 (1982).

[22] M. P. Ida And M. J. Miksis, The dynamics of thin films I: General theory, SIAM J. Appl. Math., 58 (1998), pp. 456-473.

[23] A. Indekina, I. Veretennikov, and H.-C. Chang, Front dynamics and fingering of a driven contact line, J. Fluid Mech., 373 (1998), p. 81.

[24] J. M. Jerrett and J. R. DE Bruyn, Fingering instability of a gravitationally driven contact line, Phys. Fluids A, 4 (1992), pp. 234-242.

[25] M. F. G. Johnson, R. A. Schluter, M. J. Miksis, and S. G. Bankoff, Experimental study of rivulet formation on an inclined plate by fluorescent imaging, J. Fluid Mech., 394 (1999), pp. 339-354.

[26] P. Jop, Y. Forterre, and O. Pouliquen, A constitutive law for dense granular flows, Nature, 441 (2006), pp. 727-730.

[27] B. L. Keyfitz and H. C. Kranzer, Spaces of weighted measures for conservation laws with singular shock solutions, J. Differential Equations, 118 (1995), pp. 420-451.

[28] L. Kondic And J. Diez, Pattern formation in the flow of thin films down an incline: Constant flux configuration, Phys. Fluids, 13 (2001), p. 3168.

[29] H. C. Kranzer and B. L. Keyfitz, A strictly hyperbolic system of conservation laws admitting singular shocks, IMA Volumes in Mathematics and its Applications, (1990), pp. 107-125.

[30] I. M. Krieger, Rheology of monodisperse latices, Advances in Colloid and Interface Science, 3 (1972), pp. 111-136. 
[31] G. J. KYnCh, A theory of sedimentation, Trans. Faraday Soc., 48 (1952), pp. 166-176.

[32] P. D. LAx, Hyperbolic systems of conservation laws and mathematical theory of shock waves, vol. 11 of CBMS-NSF Regional Conference Series in Applied Mathematics, SIAM, 1973.

[33] L. Léger, H. Hervet, G. Massey, and E. Durliat, Wall slip in polymer melts, J. Phys.: Condens. Matter, 9 (1997), pp. 7719-7740.

[34] D. Leighton And A. ACrivos, The shear-induced migration of particles in concentrated suspensions, J. Fluid Mech., 181 (1987).

[35] R. LeVy AND M. SheARER, The motion of a thin liquid film driven by surfactant and gravity, SIAM J. Appl. Math., 66 (2006), pp. 1688-1609.

[36] T.-P. Liu, The Riemann problem for general $2 \times 2$ conservation laws, Trans. Amer. Math. Soc., 199 (1974), pp. 898-112.

[37] — Riemann problem for general systems of conservation laws, J. Differential Equations, 18 (1975), pp. 218-234.

[38] V. Ludviksson And E. N. Lightfoot, The dynamics of thin liquid films in the presence of surface-tension gradients, Am. Inst. Chem. Engrs. J., 17 (1971), pp. 1166-1173.

[39] J. A. Moriarty and L. W. Schwartz, Dynamic considerations in the closing and opening of holes in thin liquid films, J. Colloid Interf. Sci., 161 (1993), pp. 335-342.

[40] J. F. Morris and J. F. Brady, Pressure-driven flow of a suspension: Buoyancy effects, Int. J. Multiphase Flow, 24 (1998), pp. 105-130.

[41] A. MÜNCH AND B. WAGNeR, Numerical and asymptotic results on the linear stability of a thin film spreading down a slope of small inclination, European J. Appl. Math., 10 (1999), pp. 297-318.

[42] P. R. Nott and J. F. Brady, Pressure-driven flow of suspensions: simulation and theory, J. Fluid Mech., 275 (1994).

[43] O. A. Oleinik, Discontinuous solutions of nonlinear differential equations, Amer. Math. Soc. Transl., 26 (1963), pp. 95-172.

[44] A. Oron, S. H. Davis, And S. G. Bankoff, Long-scale evolution of thin liquid films, Rev. Modern Phys., 69 (1997), pp. 931-980.

[45] R. J. Phillips, R. C. Armstrong, R. A. Brown, A. L. Graham, and J. R. Авbott, A constitutive equatioin for concentrated suspensions that accounts for shear-induced particle migration, Phys. Fluids A, 4 (1992), pp. 30-40.

[46] J. F. Richardson And W. N. ZAKi, Sedimentation and fluidization: Part I, Trans. Inst. Chem. Eng., 32 (1954), pp. 35-53.

[47] U. Schaflinger, A. Acrivos, And K. Zhang, Viscous resuspension of a sediment within a laminar and stratified flow, Int. J. Multiphase Flow, 16 (1990), pp. 567-578.

[48] M. SEver, Distribution solutions of nonlinear systems of conservation laws, Mem. Amer. Math. Soc., (2007). to appear.

[49] A. Sierou And J. F. Brady, Rheology and microstructure in concentrated noncolloidal suspensions, J. Rheology, 46 (2002), pp. 1031-1056.

[50] N. Silvi And E. B. Dussan V, On the rewetting of an inclined solid surface by a liquid, Phys. Fluids, 28 (1985), pp. 5-7.

[51] M. A. Spaid And G. M. Homsy, Stability of Newtonian and viscoelastic dynamic contact lines, Phys. Fluids, 8 (1995), pp. 460-478.

[52] J. J. Stickel and R. L. Powell, Fluid mechanics and rheology of dense suspensions, Ann. Rev. Fluid Mech., 37 (2005), pp. 129-149.

[53] H. Tang, W. Grivas, D. Homentcovschi, J. Geer, and T. Singler, Stability considerations associated with the meniscoid particle band at advancing interfaces in Hele-Shaw suspension flows, Phys. Rev. Lett., 85 (2000), pp. 2112-2115.

[54] B. D. Timberlake And J. F. Morris, Particle migration and free-surface topography in inclined plane flow of a suspension, J. Fluid Mech., 538 (2005).

[55] S. M. Troian, E. Herbolzheimer, S. A. Safran, and J. F. Joanny, Fingering instabilities of driven spreading films, Europhys. Lett., 10 (1989).

[56] M. Ungarish, Hydrodynamics of Suspensions, Springer-Verlag, 1993, ch. 2: PhysicoMathematical Formulation, pp. 7-36.

[57] T. P. Witelski And A. J. Bernoff, Dynamics of three-dimensional thin film rupture, Phys. D, 147 (2000), pp. 155-176.

[58] L. Xianfan and C. Pozrikidis, Film flow of a suspension down an inclined plane, Phil. Trans. R. Soc. Lond., 361 (2003), p. 847.

[59] J. Zhou, B. Dupuy, A. Bertozzi, And A. E. Hosoi, Theory for shock dynamics in particleladen thin films, Phys. Rev. Lett., 94 (2005). 